\title{
Non-axisymmetric dynamic response of imperfectly bonded buried liquid- filled orthotropic thin cylindrical shell due to incident shear wave (SH wave)
}

\author{
Rakesh Singh Rajput $^{1 *}$, Sunil Kumar ${ }^{2}$, Alok Chaube ${ }^{3}$, J. P. Dwivedi ${ }^{4}$ \\ ${ }^{1}$ Mechanical Engineering, Directorate of Technical Education Bhopal (M.P.) INDIA \\ ${ }^{2,3}$ Mecanical Engineering, Rajeev Gandhi Technical University, Bhopal, M. P., INDIA \\ ${ }^{4}$ Dept. of Mechanical Engineering, IT-BHU, Varanasi, INDIA \\ *Corresponding Author e-mail rak6raj@yahoo.co.in, Tel +91-755-2661885, Fax.+917552-266061
}

\begin{abstract}
The main aim of this paper is to assess the effects of the liquid presence and the bond imperfection while evaluating the nonaxisymmetric dynamic response of an imperfectly bonded liquid filled buried orthotropic thin cylindrical pipeline excited by shear horizontal wave (SH-wave) due to seismic excitation. Using thin shell theory, the effect of shear deformation and rotary inertia is not considered. The pipeline is modeled as an infinite thin cylindrical shell imperfectly bonded to surrounding. A thin layer is assumed between the shell and the surrounding medium (soil) such that this layer possesses the properties of stiffness and damping both. The degree of imperfection of the bond is varied by changing the stiffness and the damping parameters of this layer. For the wave propagation in the liquid inside the pipe, linear acoustic equation is used. The effects of the liquid presence on the shell displacement are studied for different soil condition and at various angles of incidence of the shear wave. The effect of the bond imperfection on the shell response is compared with the effects realized due to the presence of liquid inside the pipeline. It is found that magnitude of the response of liquid filled pipeline can become even more than that of an empty pipeline, and hence, it cannot be assumed that a liquid filled pipeline will always furnish safe and conservative response. Numerical results are presented for the case of an incident plane shear horizontal wave (SH- wave) only. Such studies are critical for design considerations for providing utility services through underground pipelines made of orthotropic material in seismic zones.
\end{abstract}

Keywords: Buried Pipelines, Non-axisymmetric, Imperfect Bond, Seismic Wave, Thin Shell and Shear Wave.

\section{Introduction}

Growing urbanization with increasing utility services requirement has led to increased used of underground pipes. It has necessitated the dynamic response analysis of such pipes under seismic excitation. Earlier researches have dealt with the pipes made of isotropic material. After arrival of reinforced plastic mortar (RPM) pipes need is felt to analyze the pipe of orthotropic materials. As a result, during past few years a number of studies like Cole Ritter and Jordon (1979) and Singh et al (1987) on the axisymmetric dynamic response of buried orthotropic pipe/shells are reported. Later Chonan (1981); Dwivedi and Upadhyay (1989; 1990; 1991); and Dwivedi et al (1991) have analyzed the axisymmetric problems of imperfectly bonded shell for the pipes made of orthotropic materials. Upadhyay and Mishra (1988) have presented a good account of work on non-axisymmetric response of buried thick orthotropic pipelines under seismic excitation. Again Dwivedi et al (1992a; 1992b); Dwivedi et al (1993a; 1993b; 1996); and Dwivedi et al. (1998) have analyzed the non-axisymmetric problems of imperfectly bonded buried thick orthotropic cylindrical shells. Kauretzis et al. (2007) have presented analytical calculations of blast induced strains on buried pipe lines. Hasheninajad and Kazemirad (2008) have reported dynamic response of eccentric tunnel in poro-elastic soil under sesmic excitation. Lee et al. (2009) have done the risk analysis of buried pipelines using probabilistic method. But in all these analyses pipelines are modeled as thick shell. As far as the non-axisymmetric dynamic response of thin shell is concerned, no work is reported so far. There is no work available discussing the effect of bond imperfection on the non axisymmetric response of 
buried thin pipes made of orthotropic materials. Therefore, in present paper, the effect of imperfect bond on the non- axisymmetric dynamic response of buried orthotropic thin pipelines is analyzed.

\section{Basic Equations and Formulations}

The pipeline is modeled as an infinitely long cylindrical shell of mean radius $\mathrm{R}$ and thickness $\mathrm{h}$. It is considered to be buried in a linearly elastic, homogeneous and isotropic medium of infinite extent. Basic approach of the formulation is to obtain the mid plane displacements of the shell by solving the equations of motion of the orthotropic shell. Traction terms in the equations of motion are obtained by solving the three-dimensional wave equation in the surrounding medium. Appropriate boundary conditions are applied at the shell surfaces. Equations arising out of boundary conditions along with the equations of motion of the shell are simplified to yield a response equation in matrix form.

Equation governing the non axis-symmetric motion of an infinitely long orthotropic cylinder is derived following the approach of Herrmann and Mirsky (1957), Figure 0.

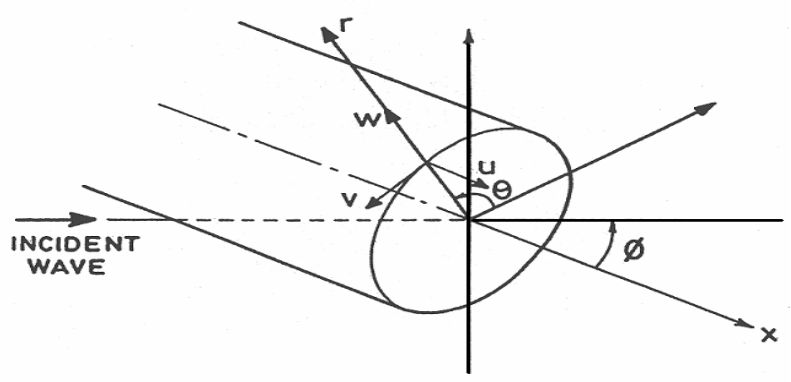

Figure 0. An infinitely long cylindrical shell

Considering an infinitely long cylindrical shell of mean radius $\mathrm{R}$ and thickness $\mathrm{h}$ buried in a linearly elastic, homogeneous and isotropic medium of infinite medium, a thin layer is assumed between the shell and the surrounding medium (soil). The degree of imperfection of the bond is varied by changing the stiffness and the damping parameters of this layer. The shell is excited by a shear horizontal wave (SH- wave). A wavelength $\Lambda(=2 \Pi / \xi)$ is considered which strikes the shell at an angle $\alpha$ with the axis of the shell. Let a cylindrical polar co-ordinate system $(\mathrm{r}, \theta, \mathrm{x})$ which is defined in such a way that $\mathrm{x}$ coincides with the axis of the shell and, in addition, $\mathrm{z}$ is measured normal to the shell middle surface, which is given as

$$
\mathrm{z}=\mathrm{r}-\mathrm{R}, \quad-\mathrm{h} / 2 \leq \mathrm{z} \leq \mathrm{h} / 2
$$

The basic equations which describe the dynamic behavior of cylindrical shells with bending resistance under arbitrary loads are derived from the system of equations which is presented by Upadhyay and Mishra (1988). But in the thin shell theory effect of shear deformation and rotary inertia is not considered. After equating all the inertial and moment terms to zero, the equilibrium equations of thick shell in stress form (from above reference) reduces to

$$
\begin{aligned}
& \frac{1}{R} \frac{\partial Q_{\theta}}{\partial \theta}+\frac{\partial Q_{x}}{\partial x}-\frac{N_{\theta \theta}}{R}+P_{1}^{*}=\rho h \frac{\partial^{2} w}{\partial t^{2}} \\
& \frac{1}{R} \frac{\partial N_{\theta \theta}}{\partial \theta}+\frac{\partial N_{x \theta}}{\partial x}+\frac{Q_{\theta}}{R}+P_{2}^{*}=\rho h \frac{\partial^{2} v}{\partial t^{2}} \\
& \frac{1}{R} \frac{\partial M_{\theta \theta}}{\partial \theta}+\frac{\partial M_{x \theta}}{\partial x}-Q_{\theta}=0 ; \\
& \frac{1}{R} \frac{\partial N_{x x}}{\partial x}+\frac{1}{R} \frac{N_{\theta x}}{\partial \theta}+P_{4}^{*}=\rho h\left[\frac{\partial^{2} u}{\partial t^{2}}\right] ; \\
& \frac{\partial M_{x x}}{\partial x}+\frac{1}{R} \frac{\partial M_{\theta x}}{\partial \theta}-Q_{x}=0
\end{aligned}
$$

In connection with the equation of equilibrium, it can be argued that transverse shearing force $Q_{\theta}$ makes a negligible contribution to equilibrium of forces in circumferential direction. So after making $Q_{\theta}$ equal to zero in Eq. $2 \mathrm{~b}$ and finding out the value of $Q_{\theta}$ and $Q_{x}$ from Eq. 2c and 2e and putting into Eq. 2b and 2d, above equations reduce to 


$$
\begin{aligned}
& \frac{\partial^{2} M_{x x}}{\partial x^{2}}+\frac{\partial^{2} M_{x \theta}}{R \partial \theta \partial x}+\frac{\partial^{2} M_{\theta x}}{R \partial \theta \partial x}+\frac{1}{R^{2}} \frac{\partial^{2} M_{\theta \theta}}{\partial \theta^{2}}-\frac{N_{\theta \theta}}{R}+P_{1}^{*}=\rho h \frac{\partial^{2} w}{\partial t^{2}} \\
& \frac{1}{R} \frac{\partial N_{\theta \theta}}{\partial \theta}+\frac{\partial N_{x \theta}}{\partial x}+P_{2}^{*}=\rho h \frac{\partial^{2} v}{\partial t^{2}} \\
& \frac{\partial N_{x x}}{\partial x}+\frac{1}{R} \frac{\partial N_{\theta x}}{\partial \theta}+P_{4}^{*}=\rho h\left\{\frac{\partial^{2} u}{\partial t^{2}}\right\}
\end{aligned}
$$

For thin shell theory shear deformation is not considered due to negligible thickness. So, according to Herrman and Mirsky (1957), the shear strain components $\gamma_{x z}$ and $\gamma_{z \theta}$ will be zero about z-axis in $\mathrm{r}-\theta$ and $\mathrm{r}$-x plane (no coupling is there due to negligible thickness) but at the same time shear stress component will be there due to Kirchhoff's hypothesis (Herrman and Mirsky, 1957)

$$
\gamma_{x z}=\frac{\partial w}{\partial x}+\psi_{x}=0 ; \gamma_{z \theta}=\frac{1}{R+z} \frac{\partial w}{\partial \theta}+\psi_{\theta}-\frac{1}{R+z}\left(v+z \psi_{\theta}\right)=0 .
$$

So from the above equations

$$
\begin{aligned}
& \psi_{x}=-\frac{\partial w}{\partial x} ; \\
& \psi_{\theta}=\frac{1}{R}\left(v-\frac{\partial w}{\partial \theta}\right) ;
\end{aligned}
$$

Here $\psi_{x}$ and $\psi_{\theta}$ are angle of rotation in $\mathrm{r}-\mathrm{x}$ and $\mathrm{r}-\theta$ plane but in the $\mathrm{r}-\theta$ plane the tangential deflection is negligible compared to component of radial deflection in that direction. So

$$
\begin{aligned}
& \psi_{x}=-\frac{\partial w}{\partial x} ; \\
& \psi_{\theta}=-\frac{1}{R}\left(\frac{\partial w}{\partial \theta}\right) ;
\end{aligned}
$$

From the above, stress resultants come out to be

$$
\begin{aligned}
& N_{x x}=E_{p} \frac{\partial u}{\partial x}-\frac{D}{R} \frac{\partial^{2} w}{\partial x^{2}}+\frac{v_{\theta x} E_{p}}{R}\left(w+\frac{\partial v}{\partial \theta}\right) ; \\
& N_{\theta x}=G_{\theta x}\left[h \frac{\partial v}{\partial x}+\frac{1}{R}\left(h+I / R^{2}\right) \frac{\partial u}{\partial \theta}+\left(I / R^{2}\right) \frac{\partial^{2} w}{\partial \theta \partial x}\right] ; \\
& M_{x x}=\frac{D}{R}\left[\frac{\partial u}{\partial x}-R \frac{\partial^{2} w}{\partial x^{2}}-\frac{v_{\theta x}}{R} \frac{\partial^{2} w}{\partial \theta^{2}}\right) ; \\
& M_{\theta x}=G_{x \theta}\left[-2(I / R) \frac{\partial^{2} w}{\partial \theta \partial x}-\left(I / R^{2}\right) \frac{\partial u}{\partial \theta}\right] ; \\
& N_{x \theta}=G_{x \theta}\left[h \frac{\partial v}{\partial x}-\left(I / R^{2}\right) \frac{\partial^{2} w}{\partial \theta \partial x}+(h / R) \frac{\partial u}{\partial \theta}\right] ; \\
& N_{\theta \theta}=\left(\frac{E_{p}^{\prime}}{R}+\frac{D^{\prime}}{R^{3}}\right)\left(w+\frac{\partial v}{\partial \theta}\right)+\frac{D^{\prime}}{R^{3}} \frac{\partial^{2} w}{\partial \theta^{2}}+v_{\theta x} E_{p} \frac{\partial u}{\partial x} ; \\
& M_{x \theta}=G_{x \theta}(I / R)\left[\frac{\partial v}{\partial x}-2 \frac{\partial^{2} w}{\partial \theta \partial x}\right] ; \\
& M_{\theta \theta}=-\frac{D^{\prime}}{R^{2}} \frac{\partial^{2} w}{\partial \theta^{2}}-\frac{D^{\prime}}{R^{2}}\left(w+\frac{\partial v}{\partial \theta}\right)-v_{\theta x} D \frac{\partial^{2} w}{\partial x^{2}} ;
\end{aligned}
$$

When we put these values of stress resultants into above equation of equilibrium, we get the required equation of motion of shell in the matrix form as

$$
[\{\mathrm{L}\}\{\mathrm{U}\}]+\left\{\mathrm{P}^{*}\right\}=0
$$


Where $[\mathrm{L}]$ is $3 \times 3$ a matrix operator with terms

$$
\begin{aligned}
L_{11}= & D \frac{\partial^{4}}{\partial x^{4}}+\frac{D^{\prime}}{R^{4}} \frac{\partial^{2}}{\partial \theta^{2}}+\frac{D^{\prime}}{R^{4}} \frac{\partial^{4}}{\partial \theta^{4}}+\frac{2 v_{\theta x} D}{R^{2}} \frac{\partial^{2}}{\partial \theta^{2} \partial x^{2}} \\
& +4 G_{x \theta}\left(\frac{I}{R^{2}}\right) \frac{\partial^{4}}{\partial \theta^{2} \partial x^{2}}+\left(\frac{E_{p}^{\prime}}{R^{2}}+\frac{D^{\prime}}{R^{4}}\right)+\rho h \frac{\partial^{2}}{\partial t^{2}} ; \\
L_{12}= & \frac{D^{\prime}}{R^{4}} \frac{\partial^{3}}{\partial \theta^{3}}-G_{x \theta}\left(\frac{I}{R^{2}}\right) \frac{\partial^{4}}{\partial \theta^{2} \partial x^{2}}+\left(\frac{E_{p}^{\prime}}{R^{2}}+\frac{D^{\prime}}{R^{4}}\right) \frac{\partial}{\partial \theta} ; \\
\mathrm{L}_{13}= & -\frac{\mathrm{D}}{\mathrm{R}} \frac{\partial^{3}}{\partial \mathrm{x}^{3}}+\frac{v_{\theta x} \mathrm{E} \mathrm{p}}{\mathrm{R}} \frac{\partial}{\partial \mathrm{x}}+\mathrm{G}_{\mathrm{x} \theta}\left(\frac{\mathrm{I}}{\mathrm{R}^{3}}\right) \frac{\partial^{3}}{\partial \theta^{2} \partial \mathrm{x}} ; \\
L_{21}= & L_{12} ; \\
L_{22}= & G_{x \theta} h \frac{\partial^{2}}{\partial x^{2}}+\left(\frac{E_{p}^{\prime}}{R^{2}}+\frac{D^{\prime}}{R^{4}}\right) \frac{\partial^{2}}{\partial \theta^{2}}-\rho h \frac{\partial^{2}}{\partial t^{2}} ; \\
L_{23}= & \frac{G_{x \theta} h}{R} \frac{\partial^{2}}{\partial \theta \partial x}+\frac{v_{\theta x} E_{p}}{R} \frac{\partial^{2}}{\partial \theta \partial x} ; \\
L_{31}= & L_{13} ; \\
L_{32}= & L_{23} ; \\
L_{33}= & \left.E_{p} \frac{\partial^{2}}{\partial x^{2}}+\left(\frac{G_{x \theta}}{R^{2}}\right)\left(h+\frac{I}{R^{2}}\right) \frac{\partial^{2}}{\partial \theta^{2}} ; \quad \text { and }\right\}=\left[\begin{array}{lll}
\mathrm{w} \quad \mathrm{v} \quad \mathrm{u}
\end{array}\right]^{\mathrm{T}}
\end{aligned}
$$

With $\mathrm{w}, \mathrm{v}$ and $\mathrm{u}$ as the displacement components of the middle surface of the shell in the radial, tangential and axial directions respectively, the elements of $\left\{\mathrm{P}^{*}\right\}$ are given by Herrman and Mirsky (1957) as:

$$
\begin{aligned}
& P_{1}^{*}=\left.\left(1+\frac{z}{R}\right) \sigma_{z z}\right|_{-h / 2} ^{h / 2}, \quad \mathrm{P}_{2}^{*}=\left.\left(1+\frac{z}{R}\right) \sigma_{z \theta}\right|_{-\mathrm{h} / 2} ^{\mathrm{h} / 2}, \\
& P_{3}^{*}=\left.z\left(1+\frac{z}{R}\right) \sigma_{z \theta}\right|_{-h / 2} ^{h / 2}, \quad \mathrm{P}_{4}^{*}=\left.\left(1+\frac{z}{R}\right) \sigma_{z x}\right|_{-\mathrm{h} / 2} ^{\mathrm{h} / 2}, \\
& P_{5}^{*}=z\left(1+\frac{Z}{R}\right) \sigma_{z x} \begin{array}{l}
h / 2 \\
-h / 2
\end{array}
\end{aligned}
$$

Where $\sigma_{\mathrm{ij}}$ denotes the stresses with their usual meaning, but for thin shell $P_{3}^{*}$ and $P_{5}^{*}$ are zero. Different constants appearing in the expressions for $\mathrm{L}_{\mathrm{ij}}$ are defined as:

$$
E_{p}=\frac{E_{x} h}{1-v_{x \theta} v_{\theta x}}, \quad E_{p}^{\prime}=\frac{E_{\theta} h}{1-v_{x \theta} v_{\theta x}}, \quad \mathrm{D}=E_{p} \frac{h^{2}}{12}, \quad \mathrm{D}^{\prime}=E_{p}^{\prime} \frac{h^{2}}{12},
$$

$\mathrm{I}=\mathrm{h}^{3} / 12$, where $E_{\mathrm{x}}, \mathrm{E}_{\theta}$ are elastic moduli, $v_{\mathrm{x} \theta}, v_{\theta \mathrm{x}}$ the Poisson ratio $\mathrm{G}_{\mathrm{x} \theta}, \mathrm{G} v_{\mathrm{xz}}$ and $\mathrm{G}_{\mathrm{z} \theta}$ the shear moduli and $\rho$ is the density of the shell material.

' $\mathrm{n}$ ' indicate the mode in circumferential direction; $\mathrm{n}=0$ represents the axisymmetric mode.

For the evaluation of $\left\{\mathrm{P}^{*}\right\}, \sigma_{\mathrm{ij}}$ at $\mathrm{z}= \pm(\mathrm{h} / 2)$ must be determined in the terms of incident and scattered field in the surrounding ground. The total displacement field in the ground is written as

$$
\mathrm{d}=\mathrm{d}^{(\mathrm{i})}+\mathrm{d}^{(\mathrm{s})}
$$

Where $\mathrm{i}$ and s represents the incident and scattered parts respectively. By solving the wave equation in the surrounding infinite medium the components of incident and scattered fields can be written as (Chonan, 1981):

$$
d_{r}^{(i)}=\left[\begin{array}{c}
\left.\left\{\gamma I_{n}^{\prime} \frac{\gamma r}{R}\right\} B_{1}+\left\{-1 \beta_{1} \delta I_{n}^{\prime} \frac{\delta r}{R}\right\} B_{3}\right] \cos n \theta \exp [i \xi(x-c t)] \\
+\left\{n \frac{R}{r} I_{n} \frac{\delta r}{R}\right\} B_{5}
\end{array}\right]
$$




$$
\begin{aligned}
& d_{\theta}^{(i)}=\left[\begin{array}{c}
\left.\left\{-n \frac{R r}{r} I_{n} \frac{\gamma r}{R}\right\} B_{1}+\left\{i n \frac{R}{r} \beta_{1} I_{n}\left(\frac{\delta r}{R}\right)\right\} B_{3}\right] \sin n \theta \exp [i \xi(x-c t)] \text { and } \\
+\left\{-\delta I_{n}^{\prime} \frac{\delta r}{R}\right\} B_{5}
\end{array}\right] \\
& d_{x}^{(i)}=\left[\left\{i \beta_{1} I_{n} \frac{\gamma r}{R}\right\} B_{1}+\left\{\delta^{2} I_{n} \frac{\delta r}{R}\right\} B_{3}\right] x \cos n \theta \exp [i \xi(x-c t)]
\end{aligned}
$$

Where $B_{1}=B_{1}^{\prime} / R, B_{3}=B_{3}^{\prime} / R^{2}$ and $B_{5}=B_{5}^{\prime} / R$. (') denotes differentiation with respect to the argument of the Bessel functions. The constants $\mathrm{B}_{1}, \mathrm{~B}_{3}$ and $\mathrm{B}_{5}$ depend on the parameters of the incident wave and may be expressed as:

$$
\begin{aligned}
& B_{1}=(-1)^{n+1}\left(i \chi \frac{A_{1}}{\varepsilon_{1}}\right), B_{3}=(-1)^{n}\left(i \chi \frac{A_{2}}{\delta \varepsilon_{2}}\right), B_{5}=(-1)^{n}\left(\chi \frac{A_{3}}{\delta}\right) \\
& d_{r}^{(s)}=\left[\left\{\gamma K_{n}^{\prime}\left(\frac{\gamma r}{R}\right)\right\} B_{2}+\left\{-i \beta_{1} \delta K_{n}^{\prime}\left(\frac{\delta r}{R}\right)\right\} B_{4}+\left\{n\left(\frac{R}{r}\right) K_{n}\left(\frac{\delta r}{R}\right)\right\} B_{6}\right] \cos n \theta \exp [i \xi(x-c t)] \\
& d_{\theta}^{(s)}=\left[\begin{array}{c}
\left.\left\{-n\left(\frac{R}{r}\right) K_{n}\left(\frac{\gamma r}{R}\right)\right\} B_{2}+\left\{i n\left(\frac{R}{r}\right) \beta_{1} K_{n}\left(\frac{\delta r}{R}\right)\right\} B_{4}\right] \sin n \theta \exp [i \xi(x-c t)] \\
+\left\{-\delta K_{n}^{\prime}\left(\frac{\delta r}{R}\right)\right\} B_{6}
\end{array}\right] \\
& d_{x}^{(s)}=\left[\left\{i \beta_{1} K_{n}\left(\frac{r r}{R}\right)\right\} B_{2}+\left\{\delta^{2} K_{n}\left(\frac{\delta r}{R}\right)\right\} B_{4}\right] \cos n \theta \exp [i \xi(x-c t)]
\end{aligned}
$$

Here $B_{2}=B_{2}^{\prime} / R, B_{4}=B_{4}^{\prime} / R^{2}$ and $\mathrm{B}_{6}=B_{6}^{\prime} / R$.

Stress field due to the incident wave can be obtained by plugging above equations into the stress-displacement relations of the medium, and are given by:

$$
\begin{aligned}
& \sigma_{r r}^{(i)}=\frac{\mu}{R}\left[\begin{array}{l}
\left\{\left(2 \varepsilon_{1}^{2}-\varepsilon_{2}^{2}\right) I_{n}\left(\frac{\gamma r}{R}\right)+2 \gamma^{2} I_{n}^{\prime \prime}\left(\frac{\gamma r}{R}\right)\right\} B_{1} \\
+\left\{-2 i \beta_{1} \delta^{2} I_{n}^{\prime \prime}\left(\frac{\gamma r}{R}\right)\right\} B_{3} \\
+2 n\left(\frac{R}{r}\right)\left\{\delta I_{n}^{\prime}\left(\frac{\delta r}{R}\right)-\left(\frac{R}{r}\right) I_{n}\left(\frac{\delta r}{R}\right)\right\} B_{5}
\end{array}\right] \cos n \theta \exp [i \xi(x-c t)] \\
& \sigma_{r \theta}^{(i)}=\frac{\mu}{R}\left[\begin{array}{l}
2 n\left(\frac{R}{r}\right)\left\{\left(\frac{R}{r}\right) I_{n}\left(\frac{\gamma r}{R}\right)-\gamma I_{n}^{\prime}\left(\frac{\gamma r}{R}\right)\right\} B_{1} \\
+2 i n \frac{R}{r} \beta_{1}\left\{\delta I_{n}^{\prime}\left(\frac{\delta r}{R}\right)-\frac{R}{r} I_{n}\left(\frac{\delta r}{R}\right)\right\} B_{3} \\
+\left\{-\delta^{2} I_{n}^{\prime \prime}\left(\frac{\delta r}{R}\right)+\delta\left(\frac{R}{r}\right) I_{n}^{\prime}\left(\frac{\delta r}{R}\right)-\left(\frac{n R}{r}\right)^{2} I_{n}\left(\frac{\delta r}{R}\right)\right\} B_{5}
\end{array}\right] \sin n \theta \exp [i \xi(x-c t)] \\
& \sigma_{r x}^{(i)}=\frac{\mu}{R}\left[\begin{array}{c}
\left.\left\{2 i \beta_{1} Y_{n}^{\prime}\left(\frac{\gamma r}{R}\right)\right\} B_{1}+\left\{\delta\left(2 \beta_{1}^{2}-\varepsilon_{2}^{2}\right) I_{n}^{\prime}\left(\frac{\delta r}{R}\right)\right\}\right] \cos n \theta \exp [i \xi(x-c t)] \\
+\left\{i n\left(\frac{R}{r}\right) \beta_{1} I_{n}\left(\frac{\delta r}{R}\right)\right\} B_{5}
\end{array}\right] \\
& \sigma_{r \theta}^{(s)}=\frac{\mu}{R}\left[\begin{array}{l}
\left\{\left(2 \varepsilon_{1}^{2}-\varepsilon_{2}^{2}\right) K_{n}\left(\frac{\gamma r}{R}\right)+2 \gamma^{2} K_{n}^{\prime \prime}\left(\frac{\gamma r}{R}\right)\right\} B_{2}+ \\
\left\{-2 i \beta_{1} \delta^{2} K_{n}^{\prime \prime}\left(\frac{\gamma r}{R}\right)\right\} B_{4}+ \\
2 n\left(\frac{R}{r}\right)\left\{\delta K_{n}^{\prime}\left(\frac{\delta r}{R}\right)-\left(\frac{R}{r}\right) K_{n}\left(\frac{\delta r}{R}\right)\right\} B_{6}
\end{array}\right] \cos n \theta \exp [i \xi(x-c t)]
\end{aligned}
$$




$$
\begin{gathered}
\sigma_{r \theta}^{(s)}=\frac{\mu}{R}\left[\begin{array}{l}
2 n\left(\frac{R}{r}\right)\left\{\left(\frac{R}{r}\right) K_{n}\left(\frac{\gamma r}{R}\right)-\gamma K_{n}^{\prime}\left(\frac{\gamma r}{R}\right)\right\} B_{2}+ \\
2 i n \frac{R}{r} \beta_{1}\left\{\delta K_{n}^{\prime}\left(\frac{\delta r}{R}\right)-\frac{R}{r} K_{n}\left(\frac{\delta r}{R}\right)\right\} B_{4}+ \\
\left\{-\delta^{2} K_{n}^{\prime \prime}\left(\frac{\delta r}{R}\right)+\delta\left(\frac{R}{r}\right) K_{n}^{\prime}\left(\frac{\delta r}{R}\right)-\left(\frac{n R}{r}\right)^{2} K_{n}\left(\frac{\delta r}{R}\right)\right\} B_{6}
\end{array}\right] \sin n \theta \exp [i \xi(x-c t)] \\
\sigma_{r x}^{(s)}=\frac{\mu}{R}\left[\begin{array}{c}
\left.\left\{2 i \beta_{1} \gamma K_{n}^{\prime}\left(\frac{\gamma r}{R}\right)\right\} B_{2}+\left\{\delta\left(2 \beta_{1}^{2}-\varepsilon_{2}^{2}\right) K_{n}^{\prime}\left(\frac{\delta r}{R}\right)\right\} B_{4}\right] \cos n \theta \exp [i \xi(x-c t)] \\
+\left\{i n\left(\frac{R}{r}\right) \beta_{1} K_{n}\left(\frac{\delta r}{R}\right)\right\} B_{6}
\end{array}\right]
\end{gathered}
$$

With the help of above equations, the stresses at the outer surface of the shell $(\mathrm{z}=\mathrm{h} / 2$ or $\mathrm{r}=\mathrm{R}+\mathrm{h} / 2)$ can be obtained. Thus $\left\{\mathrm{P}^{*}\right\}$ in Eq. (2) can be determined.

For any disturbance propagating in liquid filled inside the pipe, Linear Acoustic Equations are the continuity equation

$$
\frac{\partial \rho_{f}}{\partial t}+\bar{\nabla} \cdot\left(\rho_{f} \cdot \bar{V}_{f}\right)=0
$$

and the Euler equation of motion:

$$
\frac{\partial \bar{V}_{f}}{\partial t}+\left(\bar{V}_{f} \cdot \bar{\nabla}\right) \bar{V}_{f}=-\frac{1}{\rho_{f}} \bar{\nabla}_{p}
$$

Displacement $\mathrm{d}(\mathrm{r}, \theta, \mathrm{x}, \mathrm{t})$, at any point, satisfies the equation of motion :

$$
c_{1}^{2} \underline{\nabla}(\underline{\nabla} \cdot \underline{d})-c_{2}^{2} \underline{\nabla} \Lambda \underline{\nabla} \Lambda \underline{d}=\frac{\partial^{2}}{\partial t^{2}}(\underline{d})
$$

Where, $c_{1}=\left\{\frac{(\lambda+2 \mu)}{\rho_{m}}\right\}^{1 / 2}, c_{2}=\left\{\frac{\mu}{\rho_{m}}\right\}^{1 / 2}$ are respectively the speeds of dilatational and shear waves in the infinite medium. $\lambda$ and $\mu$ are the Lame's constant and $\rho_{\mathrm{m}}$ is the density of the medium. Change in the density due to wave prorogation is assumed to be negligible.

For getting the stresses at the inner surface of the shell, linear acoustic equation is solved for the liquid inside the shell. For the liquid inside the shell, the radial displacement $u_{r}^{(f)}$ and the pressure ' $\mathrm{p}$ ' are obtained as

$$
\begin{aligned}
& \frac{\partial u_{r}^{(f)}}{\partial t}=-A_{f} \frac{\delta_{f}}{R} I_{1}\left(\delta_{f} \frac{r}{R}\right) e^{i \xi(x-c t)} \\
& p=-\sigma_{r r}=-\rho_{f} i \xi_{c} A_{f} I_{0}\left(\delta_{f} \frac{r}{R}\right) e^{i \xi(x-c t)}
\end{aligned}
$$

Where,

$\delta_{f}=\beta \sqrt{\left(1-\left(c / c_{f}\right)^{2}\right.}$ and $c_{f}$ is the speed of the dilational wave in the liquid.

At the shell-liquid interface the continuity of the radial displacement has been assumed, i.e.,

$$
\frac{\partial w}{\partial t}=\left[\frac{\partial d_{r}^{f}}{\partial t}\right]_{r=R-h / 2}
$$

Now the mid plane displacement and slopes are assumed to be of the form; 


$$
\begin{aligned}
& w=w_{0} \operatorname{cosn} \theta \exp [\mathrm{i} \xi(\mathrm{x}-\mathrm{ct})] \\
& v=v_{0} \operatorname{sinn} \theta \exp [\mathrm{i} \xi(\mathrm{x}-\mathrm{ct})] \\
& u=u_{0} \cos n \theta \exp [\mathrm{i} \xi(\mathrm{x}-\mathrm{ct})]
\end{aligned}
$$

Plugging Eq. (12) in Eq. (2) and (11) along with the expression for $\left\{\mathrm{P}^{*}\right\}$, a set of six simultaneous algebraic equations are obtained.

Three more equations are obtained by imposing the boundary conditions at the inner and outer surfaces of the shell: i.e.,

$$
\begin{aligned}
& w=\left(d_{r}^{(i)}+d_{r}^{(s)}\right)_{r=R+h / 2} \\
& v+(h / 2) \psi_{\theta}=\left(d_{\theta}^{(i)}+d_{\theta}^{(s)}\right)_{r=R+h / 2} \\
& u+(h / 2) \psi_{x}=\left(d_{x}^{(i)}+d_{x}^{(s)}\right)_{r=R+h / 2}
\end{aligned}
$$

Boundary conditions at the outer surface of the shell $(\mathrm{r}=\mathrm{R}+\mathrm{h} / 2)$ are obtained by assuming that the shell and the continuum are joined together by a bond which is thin, elastic and inertia less. This implies that the stress at the shell-soil interface is continuous. To take the elasticity of the bond into account, the stresses in the bond are assumed proportional to relative displacements between the shell and continuum. $\mu$ shear modulus of medium and $\rho$ density of shell material.

$$
\begin{aligned}
& \left(\sigma_{r x}\right)_{r=R+h / 2}=\left[\left(S_{x}+Z_{x} \frac{\partial}{\partial t}\right)\left(\mu_{x}^{i}+\mu_{x}^{s}-u-(r-R) \psi_{x}\right]_{r=R+h / 2}\right. \\
& \left(\sigma_{r r}\right)_{r=R+h / 2}=\left[\left(S_{r}+Z_{r} \frac{\partial}{\partial t}\right)\left(\mu_{r}^{i}+\mu_{r}^{s}-w\right)\right]_{r=R+h / 2} \\
& \left(\sigma_{r \theta}\right)_{r=R+h / 2}=\left[\left(S_{\theta}+Z_{\theta} \frac{\partial}{\partial t}\right)\left(\mu_{\theta}^{i}+\mu_{\theta}^{s}-u(r-R) \psi_{\theta}\right]_{r=R+h / 2}\right. \\
& \zeta_{R}=\frac{\mu}{S_{r} \cdot R}, \zeta_{\theta}=\frac{\mu}{S_{\theta} \cdot R} \text { and } \zeta_{x}=\frac{\mu}{S_{x} \cdot R} \text { are the non dimensionalized stiffness coefficient of the bond in radial, tangential }
\end{aligned}
$$

and axial direction respectively.

$$
\Gamma_{r}=\frac{\mu}{Z_{r} c_{1}} \Gamma_{\theta}=\frac{\mu}{Z_{\theta} c_{1}} \text { and } \Gamma_{x}=\frac{\mu}{Z_{x} c_{1}} \text { are the non dimensionalized damping coefficient of the bond in radial , }
$$
tangential and axial direction respectively.

Thus a total of seven algebraic equations are obtained. These seven equations when simplified give the final response equation, which may be put into the form

$$
\{Q\}\left\{U_{0}\right\}=\mathrm{B}_{1}\left\{\mathrm{~F}^{1}\right\}+\mathrm{B}_{3}\left\{F^{2}\right\}+\mathrm{B}_{5}\left\{F^{3}\right\}
$$

Where $[\mathrm{Q}]$ is a $(7 \times 7))$ matrix and $\left\{\mathrm{F}^{1}\right\},\left\{\mathrm{F}^{2}\right\}$ and $\left\{\mathrm{F}^{3}\right\}$ are $(7 \times 1)$ matrices. But for the response of horizontal shear wave the amplitudes due to shear waves $\mathrm{B}_{1}$ and $\mathrm{B}_{3}$ would be zero so the effect of $\left\{\mathrm{F}^{2}\right\}$ and $\left\{\mathrm{F}^{1}\right\}$ matrices would be eliminated. Putting values of $B_{1}=B_{3}=0$ and substituting values of $B_{5}$ from Eq. (8) Eq. (13) becomes as

$$
\{Q\}\left\{U_{0}\right\}=(-1)^{n}\left(\chi \frac{A_{3}}{\varepsilon_{3}}\right)\left\{\mathrm{F}^{3}\right\}
$$

Here it must be pointed out that for an incident P-wave $\epsilon_{1}=\beta$ whereas, for an incident shear wave (SV- or SH- wave) $\epsilon_{2}=\beta$. In the present work the non-dimensional wave number of the incident wave, i.e. $\beta(=2 \pi \mathrm{R} / \wedge)$ is given as input, so either $\epsilon_{1}$ or $\epsilon_{2}$ is always known. The other $\epsilon_{\mathrm{i}}$ can be obtained by using the following relations:

$$
\left(\frac{\epsilon_{2}}{\epsilon_{1}}\right)^{2}=\frac{\mathrm{c}_{1}^{2}}{\mathrm{c}_{2}^{2}}=\frac{2\left(1-v_{\mathrm{m}}\right)}{\left(1-2 v_{\mathrm{m}}\right)}
$$

Where, $v_{\mathrm{m}}$ is the Poisson ratio of the medium.

\section{Results and Discussions}

To study the effects of the liquid presence inside the pipe on the thin pipe displacement under different soil conditions at various angles of incidence of the shear wave under imperfect bonding, parametric results in graphical forms are generated.

Results are presented for a transversely isotropic shell with $\mathrm{r}-\theta$ as the plane of isotropy leading to $E_{\theta}=E_{z}, G_{x z}=G_{x \theta}$, $v_{x \theta}=v_{x z}, v_{\theta z}=v_{z \theta}, G_{z \theta}=E_{\theta} / 2\left(1+v_{\theta z}\right), \eta_{3}=\eta_{2}$ and $\eta_{4}=G_{z \theta} / E_{x}=\eta_{1} / 2\left(1+v_{\theta z}\right)$. In addition $v_{\theta z}=v_{x \theta}=0.3$ is taken in the 
numerical calculations. Shell orthotropy parameters $\eta_{1}$ and $\eta_{2}$ are taken as $0.5,0.1,0.05$ and $0.1,0.05,0.02$ respectively. Soil hardness parameter $\bar{\mu}$ is varied from 0.1 to 10.0 to take into account different soil conditions around the pipe. $\bar{\mu}=0.01$ corresponds to soft soil, $\bar{\mu}=0.1$ corresponds to the medium hard soil whereas $\bar{\mu}=10.0$ represent hard and rocky surroundings. For all the values of $\bar{\mu}$, Poisson ratio for pipe material $\left(v_{m}\right)$ is assumed as 0.25 . Thickness to radius ratio of the shell $(\bar{h})$ is taken as 0.01 and the density ratio of the surrounding medium to the shell $(\bar{\rho})$ is taken as 0.75 . Non-dimensional amplitudes of the middle surface of the shell in the radial and axial directions $(\bar{W}$ and $\bar{U})$ are plotted against the non-dimensional wave number of the incident SH-wave $(\beta=2 \pi \mathrm{R} / \Lambda)$. The shell response is shown for empty and liquid filled shell for non-axisymmetric mode (Flexural mode, $\mathrm{n}=1)$ taking stiffness coefficient $\left(\zeta_{x} \zeta_{r}\right)$, damping coefficient $\left(\Gamma_{x} \Gamma_{r}\right)$ as parameters.

Figures 1 to 3 show the effects of stiffness coefficient $\zeta_{r}$. With the soft surrounding soil, the radial displacement of liquid filled pipe is negligible at small angle of incidence of SH wave as compared to empty pipe. Shell orthotropy parameters are also effecting the radial displacement significantly.

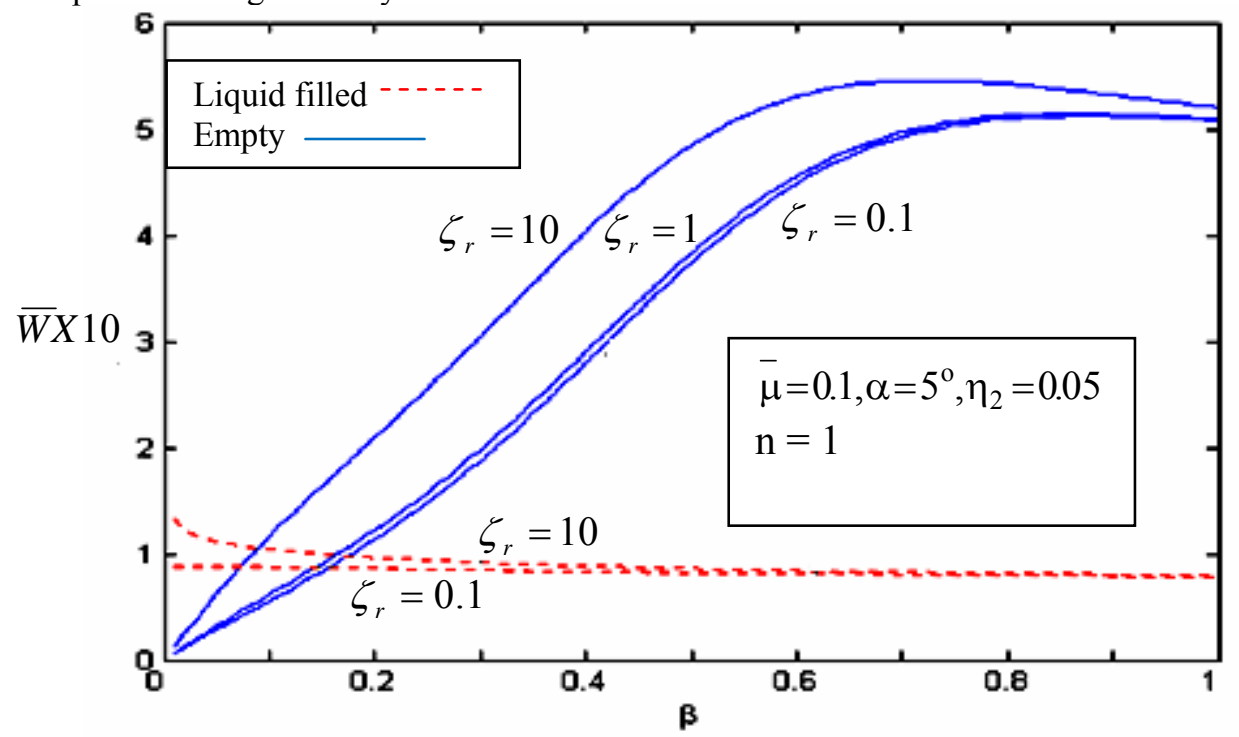

Figure1. Radial displacement $(\bar{W})$ vs. wave number $(\beta)$ with stiffness coefficient $\left(\zeta_{r}\right)$ as parameter

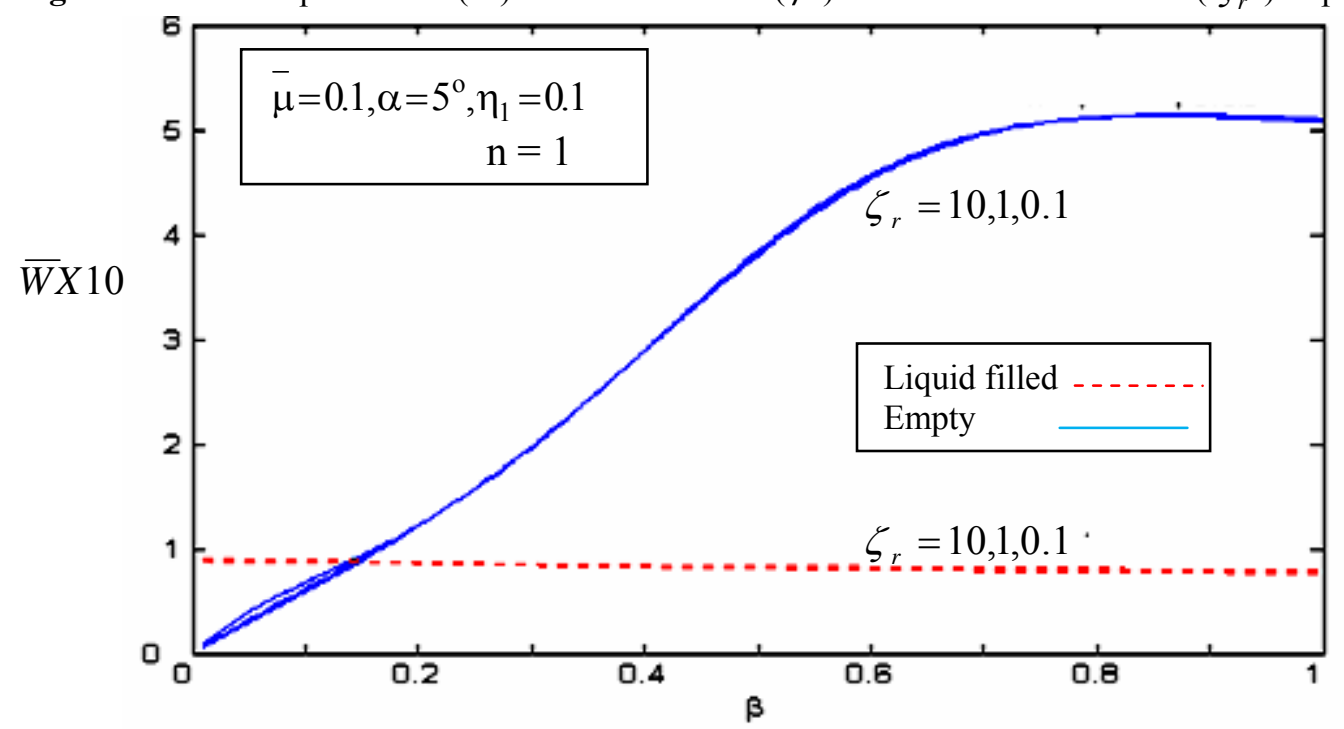

Figure 2. Radial displacement $(\bar{W})$ vs. wave number $(\beta)$ with stiffness coefficient $\zeta_{r}=0.1,1,10$ as parameter 


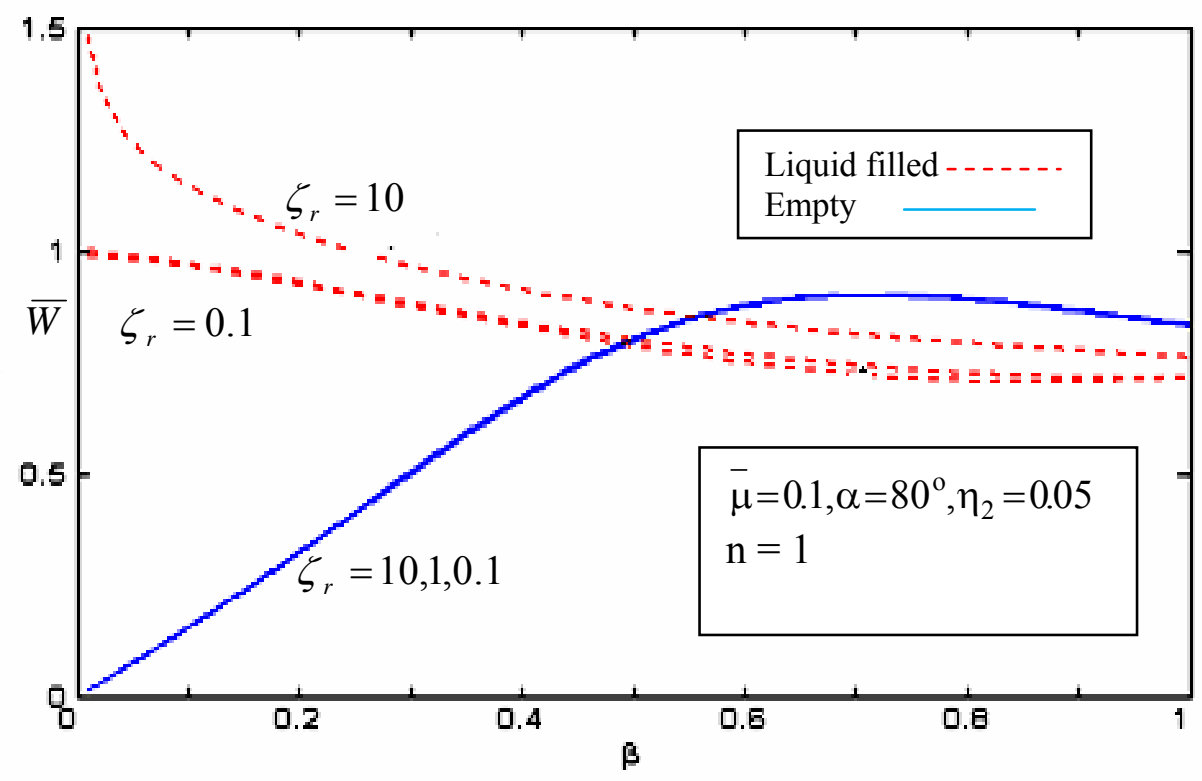

Figure 3. Radial displacement $(\bar{W})$ vs. wave number $(\beta)$ with stiffness $\zeta_{r}=0.1,1,10$ as parameter

Figures 4 to 6 show the effect of damping coefficients $(\Gamma)$ on radial $(\bar{W})$ displacement of the shell at different values of orthotropic parameters $\eta_{1}$ and $\eta_{2}$ of the shell material and the different conditions of the soil $(\bar{\mu})$ at different angle of incidence of the wave number. $(\bar{\beta})$. For larger angle of incidence radial deflection is higher in liquid filled pipe and it decreases with increasing wave number. In empty pipe, radial deflection increases with increasing wave number. Under hard rocky surrounding, the radial displacement is comparable for empty and liquid filled pipe at higher wave number. The impact of bonding parameter on radial displacement is insignificant in both the cases i.e. empty as well as liquid filled.

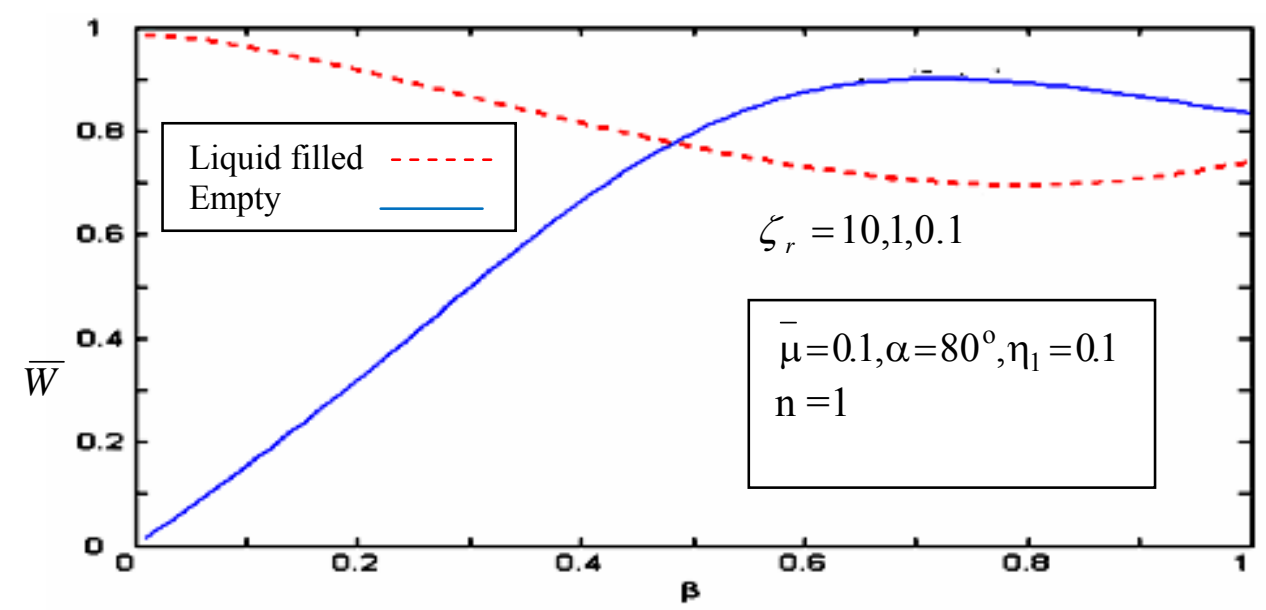

Figure 4. Radial displacement $(\bar{W})$ vs. wave number $(\beta)$ with damping coefficient $\Gamma_{r}=0.1,1,10$ as parameter 


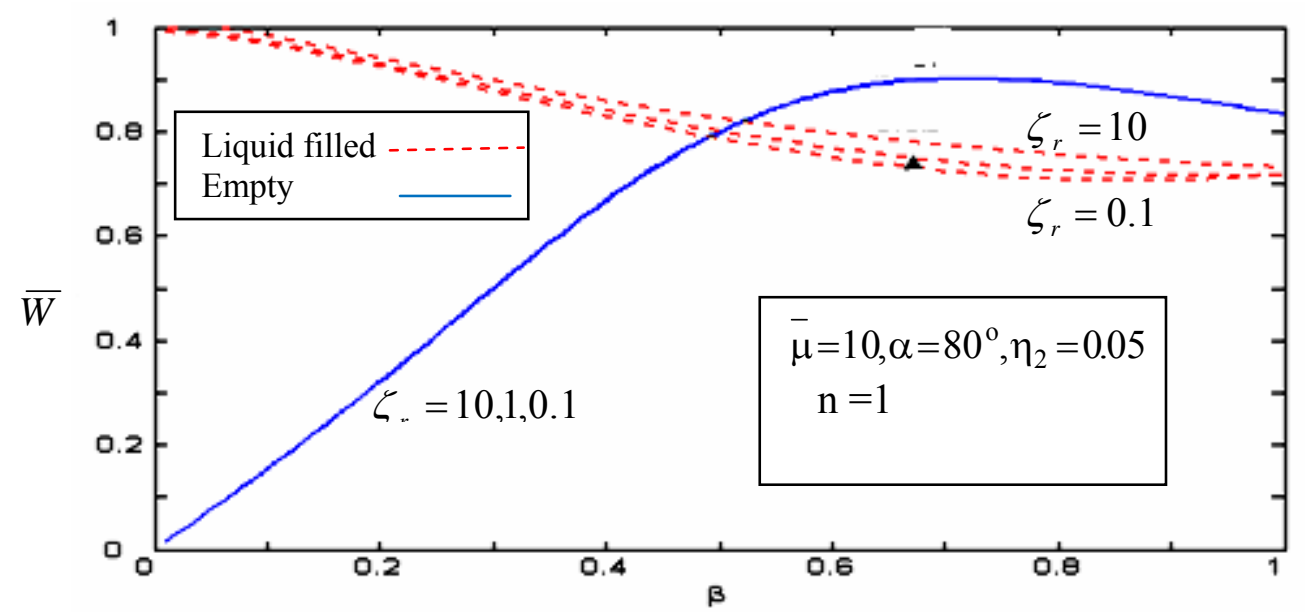

Figure 5. Radial displacement $(\bar{W})$ vs. wave number $(\beta)$ with damping coefficient $\Gamma_{r}=0.1,1,10$ as parameter

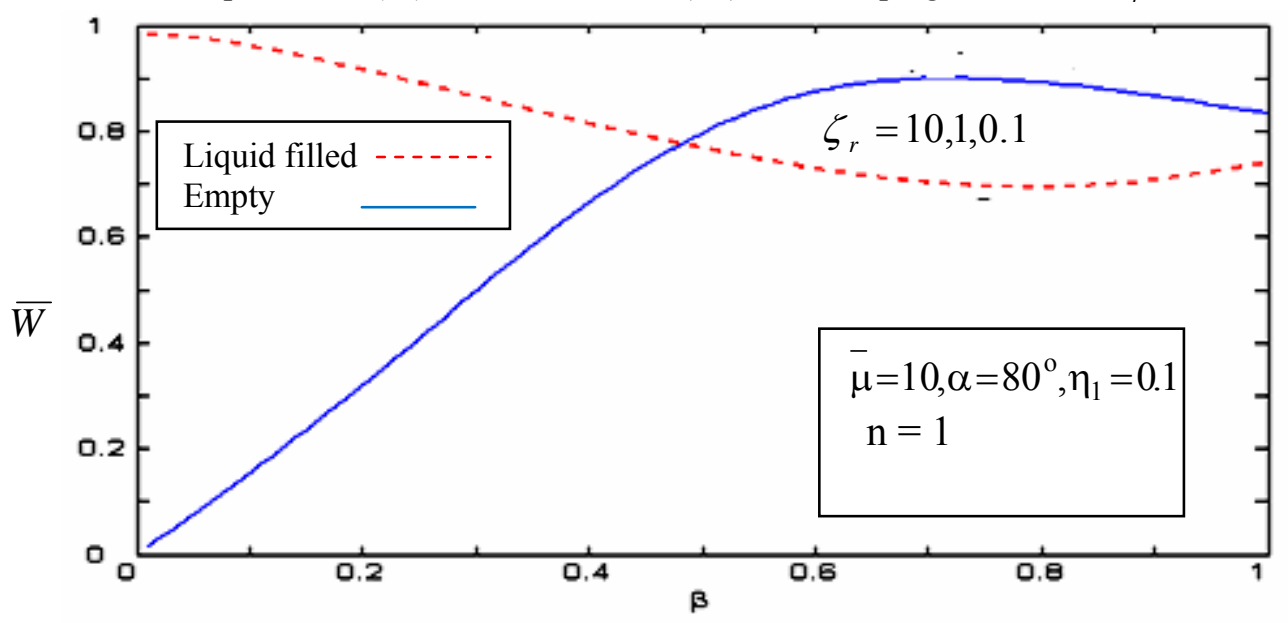

Figure 6 Radial displacement $(\bar{W})$ vs. wave number $(\beta)$ with $\Gamma_{r}=0.1,1,10$ as parameter

Figures 7 to 9 show the effects of stiffness coefficient $\zeta_{x}$ on axial $(\bar{U})$ displacement of the shell at different values of orthotropic parameters $\eta_{1}$ and $\eta_{2}$ of the shell material and the different conditions of the soil $(\bar{\mu})$ at different angle of incidence of the wave number $(\bar{\beta})$. At higher wave number, the liquid presence has negligible effect on axial displacement as compared to empty pipe at low angle of incidence. At higher angle of incidence, the axial displacement is higher in case of liquid filled pipe as compared to empty pipe with increasing wave number. In hard and rocky surrounding media and with a higher angle of incidence, the bond parameters play an important role in the axial direction dynamic response of empty pipe as compared to the response of liquid filled pipe. 


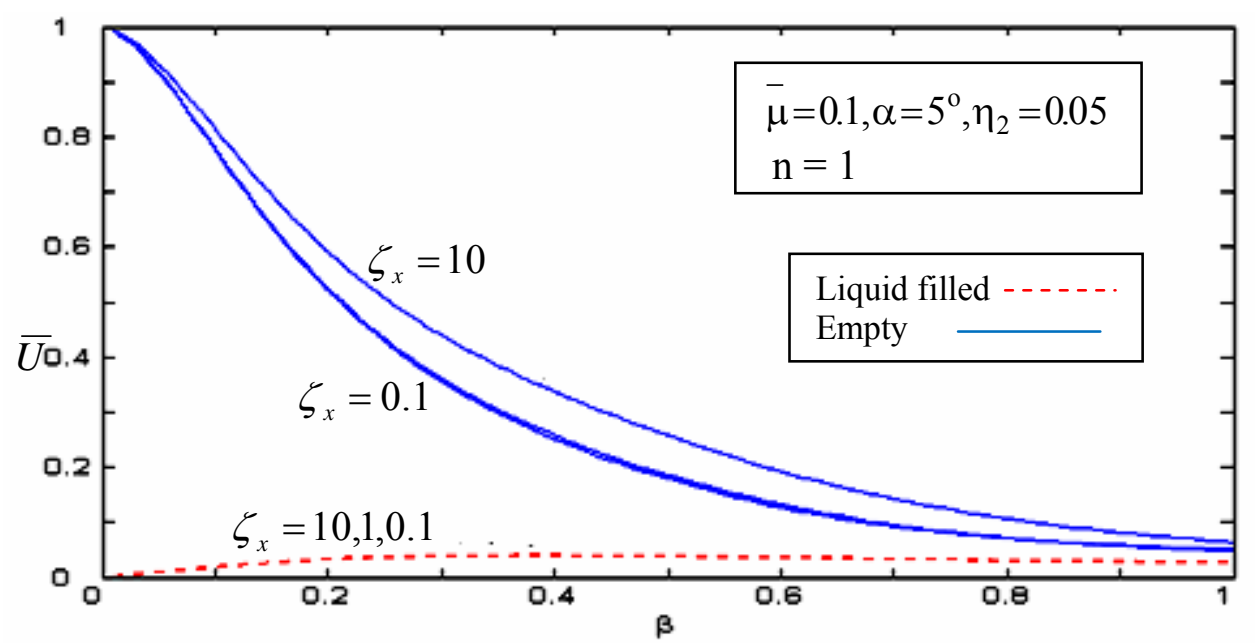

Figure 7. Axial displacement $(\bar{U})$ vs. wave number $(\beta)$ with stiffness coefficient $\zeta_{x}=0.1,1,10$ as parameter

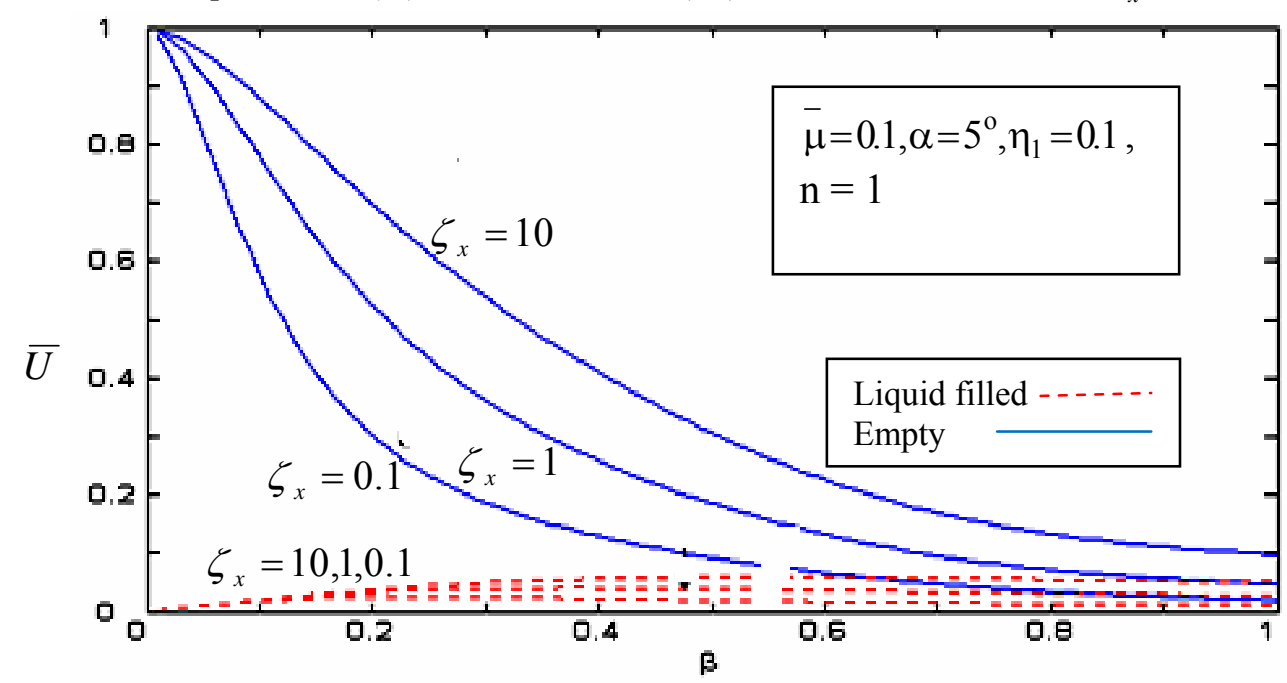

Figure 8. Axial displacement $(\bar{U})$ vs. wave number $(\beta)$ with stiffness coefficient $\zeta_{x}=0.1,1,10$ as parameter

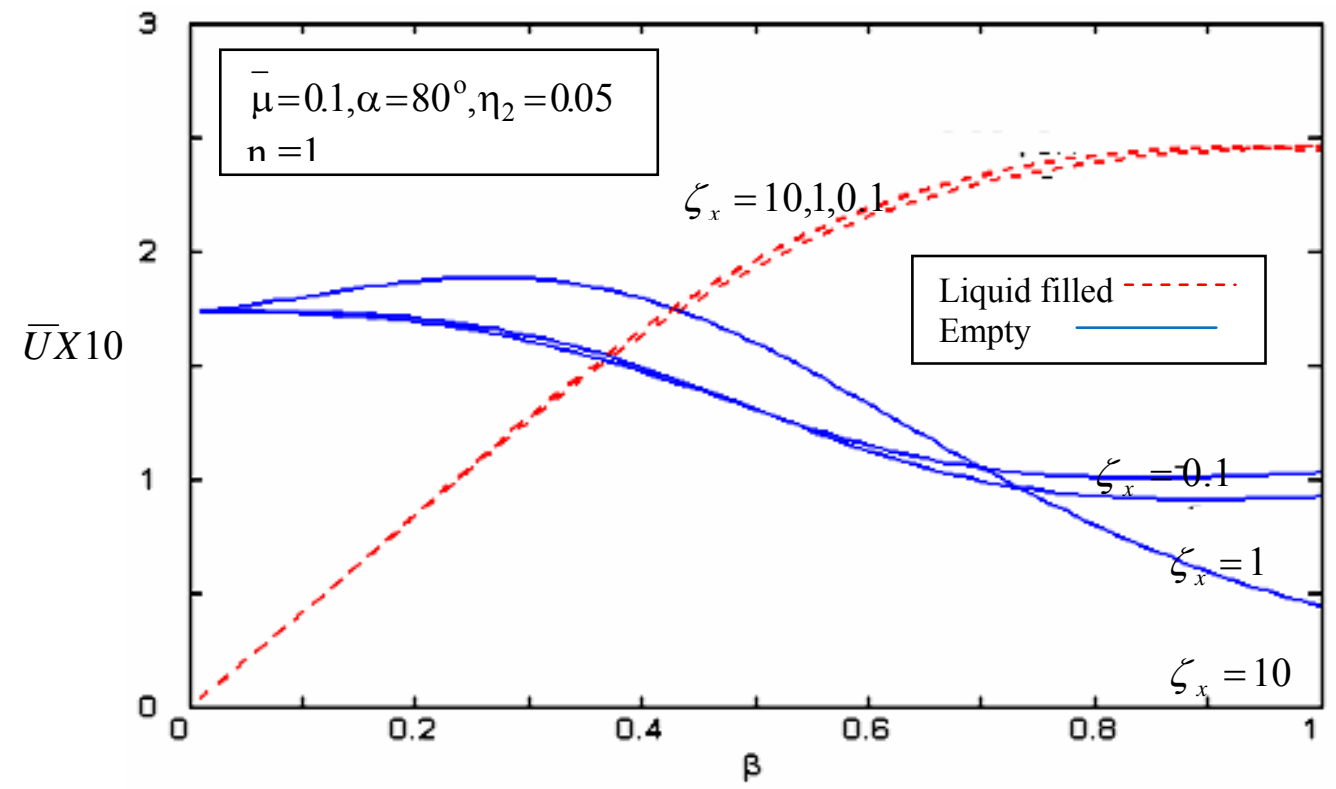

Figure 9. Axial displacement $(\bar{U})$ vs. wave number $(\beta)$ with stiffness coefficient $\zeta_{x}=0.1,1,10$ as parameter 
Figure 10 shows the effect of damping coefficients $(\Gamma)$ on axial $(\bar{U})$ displacement of the shell at different values of orthotropic parameter $\eta_{1}$ of the shell material and surrounded with soft soil $(\bar{\mu}=0.1)$ at high angle of incidence with increasing wave number $(\bar{\beta})$. It is found that magnitude of axial deflection of liquid filled pipeline can become substantially higher than that of an empty pipeline under soft soil surroundings, and hence, it cannot be assumed that a liquid filled pipeline will always furnish safe and conservative dynamic response.

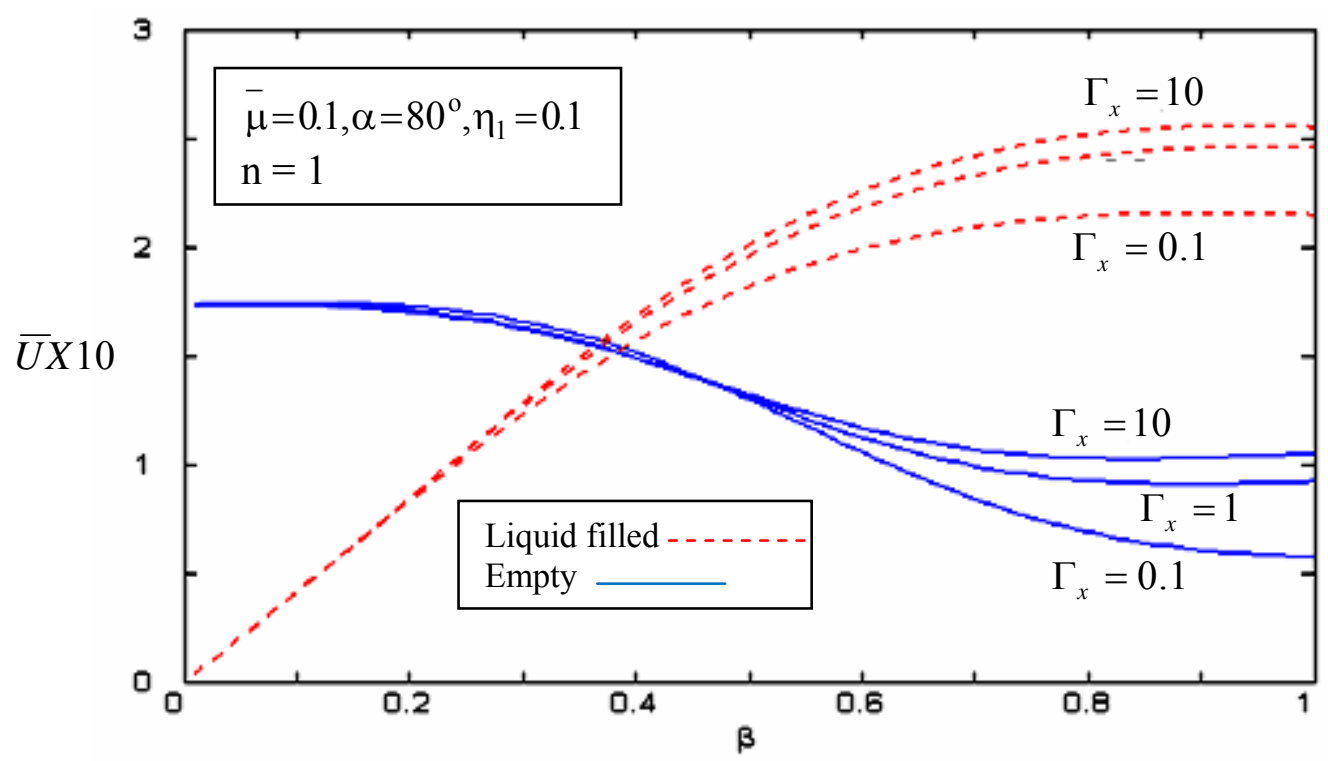

Figure 10. Axial displacement $(\bar{U})$ vs. wave number $(\beta)$ with damping coefficient $\Gamma_{x}=0.1,1,10$ as parameter

\section{Conclusions}

The effects of the liquid presence on the buried thin orthotropic pipe displacement are studied for different surrounding soil conditions under shear horizontal wave generated due to seismic excitation striking at various angles of incidence. Based on the presented results following general conclusions could be drawn:

- With the soft surrounding soil, the radial displacement of liquid filled pipe is negligible at small angle of incidence of SH wave as compared to empty pipe. Shell orthotropy parameters are also effecting the radial displacement significantly.

- In the soft surrounding soil, at higher angle of incidence of SH wave the initial displacement of the liquid filled shell higher and it decreases with increasing wave number at higher angle of incidence of SH wave. In empty pipe, radial deflection increases with increasing wave number.

- Under hard rocky surrounding, the radial displacement is comparable for empty and liquid filled pipe at higher wave number. The impact of bonding parameter on radial displacement is insignificant in both the cases i.e. empty as well as liquid filled.

- In hard and rocky surrounding media and with a higher angle of incidence, the bond parameters play an important role in the axial direction dynamic response of empty pipe as compared to the response of liquid filled pipe.

- It is observed that magnitude of axial deflection of liquid filled pipeline can become substantially higher than that of an empty pipeline under soft soil surroundings, and hence, it cannot be assumed that a liquid filled pipeline will always furnish safe and conservative dynamic response.

\section{Nomenclature}

$\mathrm{A}$

$A_{1} ; A_{2} ; A_{3}$

$\mathrm{B}_{1} \ldots \ldots, \mathrm{B}_{6}$
Amplitude of the plane wave

Amplitudes of P-SV-SH wave respectively

Arbitrary constants 


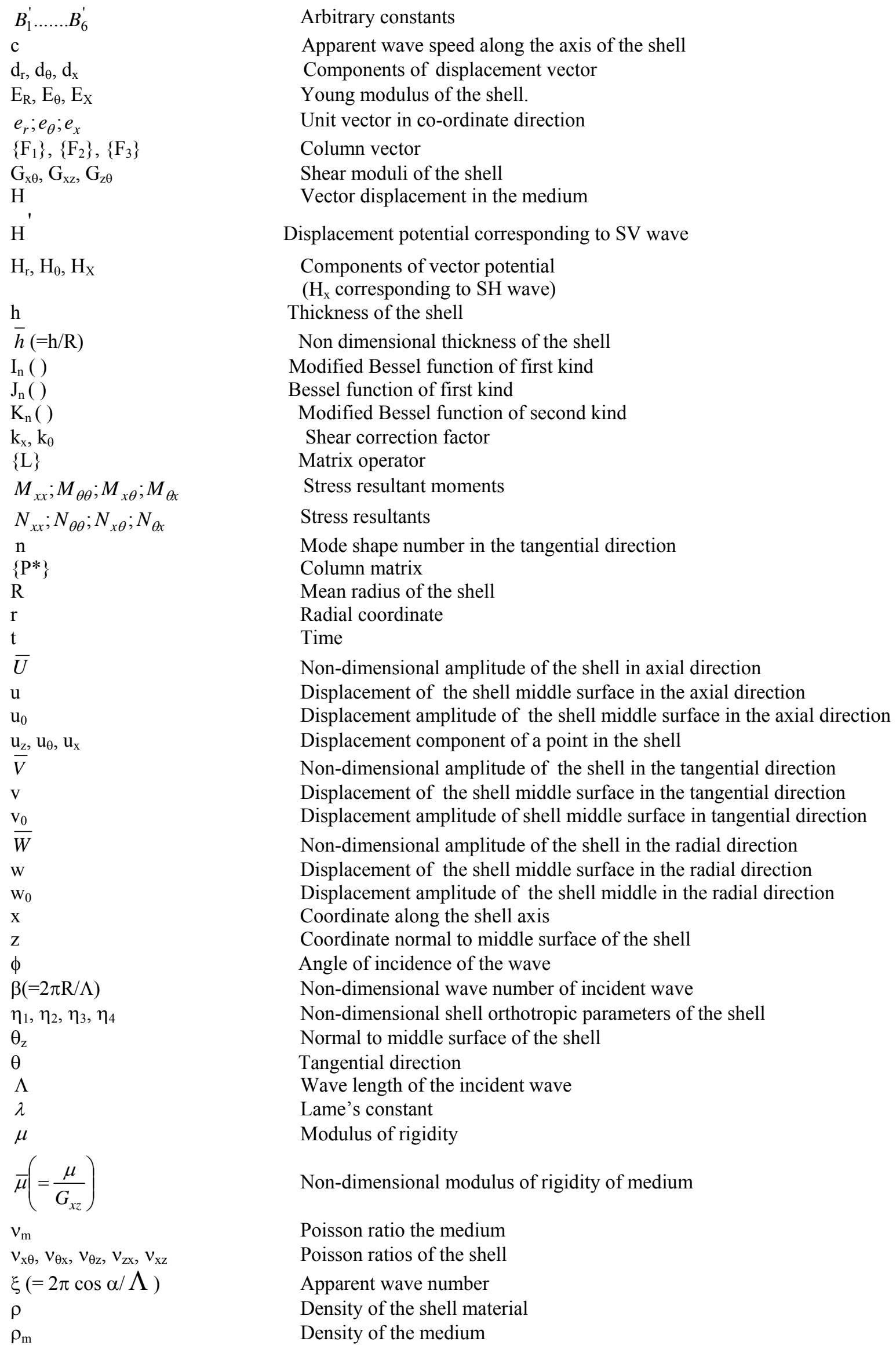

Arbitrary constants

Apparent wave speed along the axis of the shell

Components of displacement vector

Young modulus of the shell.

Unit vector in co-ordinate direction

Column vector

Shear moduli of the shell

Vector displacement in the medium

Displacement potential corresponding to SV wave

Components of vector potential

( $\mathrm{H}_{\mathrm{x}}$ corresponding to $\mathrm{SH}$ wave)

Thickness of the shell

Non dimensional thickness of the shell

Modified Bessel function of first kind

Bessel function of first kind

Modified Bessel function of second kind

Shear correction factor

Matrix operator

Stress resultant moments

Stress resultants

Mode shape number in the tangential direction

Column matrix

Mean radius of the shell

Radial coordinate

Time

Non-dimensional amplitude of the shell in axial direction

Displacement of the shell middle surface in the axial direction

Displacement amplitude of the shell middle surface in the axial direction

Displacement component of a point in the shell

Non-dimensional amplitude of the shell in the tangential direction

Displacement of the shell middle surface in the tangential direction

Displacement amplitude of shell middle surface in tangential direction

Non-dimensional amplitude of the shell in the radial direction

Displacement of the shell middle surface in the radial direction

Displacement amplitude of the shell middle in the radial direction

Coordinate along the shell axis

Coordinate normal to middle surface of the shell

Angle of incidence of the wave

Non-dimensional wave number of incident wave

Non-dimensional shell orthotropic parameters of the shell

Normal to middle surface of the shell

Tangential direction

Wave length of the incident wave

Lame's constant

Modulus of rigidity

Non-dimensional modulus of rigidity of medium

Poisson ratio the medium

Poisson ratios of the shell

Apparent wave number

Density of the shell material

Density of the medium 
$\bar{\rho}\left(=\frac{\rho_{m}}{\rho}\right)$

$\sigma_{\mathrm{ij}}$

$\phi$

$\psi_{\mathrm{x}}$

$\chi$

$\psi_{\text {хо }}$

$\psi_{\theta}$

\section{Subscripts}

$\mathrm{m}$

$\mathrm{r}$

$\mathrm{x}$

$\mathrm{Z}$

$\theta$

\section{Superscripts}

i

S

\section{References}

Ariman, T. and Muleski, G. E. 1988. Review of the response of buried pipelines under seismic Excitation. International Journal of Earthquake Engineering and Structural Dynamics, Vol. 9, pp. 133-151.

Chonan, S. 1981. Response of a pre-stressed, orthotropic thick cylindrical shell subjected to pressure pulses. Journal of Sound and Vibration, Vol. 78, pp. 257-267.

Cole, B. W., Ritter, C. J. and Jordon, S. 1979. Structural analysis of buried reinforced plastic mortar pipe. Lifeline earthquake engineering- buried pipelines, seismic risk and instrumentation, T. Ariman, S. C. Liu and R. E. Nickell, eds., ASME.

Ariman, T., Liu, S. C., and Nickell, R. E. (Eds.), Life Earthquake Engineering Buried Pipelines, Seismic Risk and Instrumentation New York: ASME, pp. 114-131.

Dwivedi, J. P. and Upadhyay, P. C. 1989. Effect of imperfect bonding on the axisymmetric dynamic response of buried orthotropic cylindrical shells. Journal of Sound and Vibration, Vol. 135, pp. 477-486.

Dwivedi, J. P. and Upadhyay, P. C. 1990. Effect of liquid presence on the dynamic response of imperfectly bonded buried orthotropic cylindrical shells. Journal of Sound and Vibration, Vol. 139, No. II, pp. 99-110.

Dwivedi, J. P. and Upadhyay, P. C. 1991. Effect of imperfect bond on the dynamic response of buried orthotropic cylindrical shells under shear-wave excitation. Journal of Sound and Vibration, Vol. 145, No. 2, pp. 333-337.

Dwivedi, J. P., Singh, V. P. and Upadhyay, P. C. 1991. Effect of fluid presence on the dynamic response of imperfectly bonded cylindrical shells due to incident shear-wave excitation. Computer and Structures, Vol. 40, No. 4, pp. 995-1001.

Dwivedi, J. P., Mishra, B. K. and Upadhyay, P. C. 1992. Non-axisymmetric dynamic response of imperfectly bonded buried orthotropic pipeline due to an incident shear wave. Journal of Sound and Vibration,Vol. 157, No. 1, pp. 81-92.

Dwivedi, J. P., Mishra, B. K. and Upadhyay, P. C. 1992b. Non-axisymmetric dynamic response of imperfectly bonded buried orthotropic pipelines due to incident shear wave (SH-wave). Journal of Sound and Vibration, Vol. 157, No. 1, pp. 177-182.

Dwivedi, J. P., Singh, V. P. and Upadhyay, P. C. 1993a. Non-axisymmetric dynamic response of imperfectly bonded buried fluidfilled orthotropic cylindrical shells due to incident shear wave. Journal of Sound and Vibration, Vol. 167, No. 2, pp. $277-287$.

Dwivedi, J. P., Singh, V. P. and Upadhyay, P. C. 1993b. Effect of fluid presence on the non-axisymmetric dynamic response of imperfectly bonded buried orthotropic pipelines due to incident shear wave. Computer and Structures, Vol. 48, No. 2, pp. 219226.

Dwivedi, J. P., Singh, V. P. and Upadhyay, P. C. 1996. Non-axisymmetric dynamic response of imperfectly bonded buried fluidfilled orthotropic cylindrical shells. ASME Journal of Pressure Vessel Technology, Vol. 118, No. 1, pp. 64-73.

Dwivedi, J. P., Mishra, B. K. and Upadhyay, P. C.1998. Non-axisymmetric dynamic response of imperfectly bonded buried orthotropic pipelines. Structural Engineering and Mechanics, Vol. 6, No. 3, pp. 291-304.

Hasheminajad, S. M. and Kazemirad, S. 2008. Dynamic response of an eccentrically lined circular tunnel in poroelastic soil under seismic excitation. Soil Dynamics and Earthquake Engineering, Vol. 28, pp. 277-292.

Herrman, G. and Mirsky, J. 1957. Non axially Symmetric Motion of Cylindrical Shell. Journal of the Acoustical Society of America, Vol 29, No. 10, pp. 1116-1123.

Jones, R.M., 1975. Mechanics of Composite Materials. McGraw Hill Publications, New-York. 
Kouretzis G. P., Bouckovalas G. D. and Gantes C. J. 2007. Analytical calculation of blast-induced strains on buried pipe lines. International Journal of Impact Engineering, Vol. 34, pp. 1683-1704.

Lee, D. H., Kim, B. H., Lee, H. and Kong, J. S. 2009. Seismic behavior of buried gas pipelines under earthquake excitation. Engineering Structure, 31, pp. 1011-1023.

Muleski, G. E., Ariman, T. S. and Ariman, C. P. 1979. A Shell Model of a Buried Pipe in a Seismic environment. ASME Journal of Pressure Vessel Technology, Vol. 101, pp. 44-50.

Upadhyay P. C. and Mishra B. K., 1988. Non-axisymmetric dynamic response of buried orthotropic cylindrical shell due to incident shear waves. Journal of sound and Vibration, Vol. 121, pp. 227-239.

Upadhyay P. C. and Mishra B. K.,1988. Non Axisymmetric Dynamic Response of buried Orthotropic shells. Journal of Sound and Vibration, Vol. 121, pp. $149-160$.

Singh, V. P., Upadhyay, P. C. and Kishore, B. 1987. On the dynamic response of Buried Orthotropic Cylindrical Shells. Journal of Sound and Vibration, Vol. 113, pp. 101-115.

Biographical notes

Rakesh Singh Rajput received M. Tech. from NIT Allahabad, India and pursuing Ph.D. from Rajeev Gandhi Technological University, Bhopal, India. He is a Reader in Mechanical Engineering, and working with Department of Technical Education (DTE), Govt. of M. P., India. His research interests include machine design and mechanics. He has written book on Engineering Mechanics.

Dr. Sunil Kumar is a Professor of Mechanical Engineering, Rajeev Gandhi Technological University, Bhopal, India. He has received M. Tech from IT-BHU, Varanasi, India and Ph. D. from IIT Delhi, India. He has more than 20 years of experience in teaching and research. His current area of research includes Combustion mechanics and System Dynamics.

Dr. Alok Chaube is a Professor of Mechanical Engineering, Rajeev Gandhi Technological University, Bhopal, India. He has received M. Tech from IIT Delhi, India and $\mathrm{Ph}$. D. from IIT Roorkee, India. He has more than 20 years of experience in teaching and research. His current area of research includes Fluid mechanics and System Dynamics.

Dr. J. P. Dwivedi is a Professor of Mechanical Engineering at IT-BHU, Varanasi, India. He has received M. Tech and Ph. D. from IT-BHU, Varanasi, India. He has more than 30 years of experience in teaching and research. His current area of research includes Engineering Mechanics, Fluid mechanics and System Design.

Received October 2010

Accepted December 2010

Final acceptance in revised form December 2010 